\title{
Inhibición de Enterobacter cloacae por Bifidobacterium longum
}

\author{
Inhibition of Enterobacter cloacae by Bifidobacterium longum
}

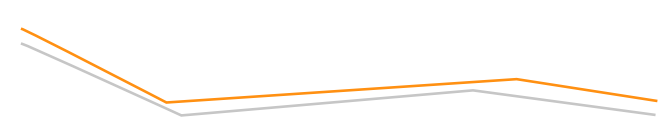

María Porfiria Barrón-González*® ${ }^{*}$ Carlos Mireles-Rangel*, Fabiola Lizeth Cuéllar-Guevara*, Daniel J. Eguiarte-Lara**, Yadira Quiñones-Gutiérrez*

Barrón-González, M. P., Mireles-Rangel, C., Cuéllar-Guevara, F. L., Eguiarte-Lara, D. J., \& Quiñones-Gutiérrez, Y. (2019). Inhibición de Enterobacter cloacae por Bifidobacterium longum. Investigación y Ciencia de la Universidad Autónoma de Aguascalientes, 27(77), 19-26.

\section{RESUMEN}

Enterobacter cloacae forma parte de la microbiota intestinal en humanos, pertenece al phylum de las Proteobacterias, familia Enterobacteriaceae, género Enterobacter, especie E. cloacae; se caracteriza por ser ubicua, oportunista, fármaco-resistente y responsable de bacteriemias e infecciones nosocomiales, por lo que se hace necesaria la búsqueda de alternativas para su tratamiento y control. En este trabajo se muestra evidencia de la inhibición del crecimiento de E. cloacae empleando metabolitos bacterianos o sobrenadantes libres de células (también conocidos como postbióticos) producidos por Bifidobacterium longum (probiótico que pertenece al filo Actinobacteria); varios trabajos demuestran la importancia de estos para

Palabras clave: Bifidobacterium longum; Enterobacter cloacae; probióticos; postbióticos; microbiota; interferencia microbiana.

Keywords: Bifidobacterium longum; Enterobacter cloacae; probiotics; postbiotics; microbiota; microbial interference.

Recibido: 11 de mayo de 2018, aceptado: 4 de marzo de 2019

* Departamento de Biología Celular y Genética, Facultad de Ciencias Biológicas, Universidad Autónoma de Nuevo León. Pedro de Alba S/N cruz con Av. Manuel L. Barragán, Ciudad Universitaria, C. P. 66455, Nuevo León, México. Correo electrónico: maria.barrongn@uanl.edu.mx; miraca010176@gmail.com; fabiola.cuellar13@gmail.com; yadiragtz70@ hotmail.com. ORCID: http://orcid.org/0000-0002-8386-444X; http:// orcid.org/0000-0003-2621-8887; http://orcid.org/0000-0001-9993-1830; http://orcid.org/0000-0002-7608-4175

** Departamento de Microbiología e Inmunología, Facultad de Ciencias Biológicas, Universidad Autónoma de Nuevo León. Pedro de Alba S/N cruz con Av. Manuel L. Barragán, Ciudad Universitaria, C. P. 66455 Nuevo León, México. Correo electrónico: daneguiarte@yahoo.com.mx. ORCID: http://orcid.org/0000-0002-4342-4175

$凶$ Autor para correspondencia inhibir el crecimiento de diversos microorganismos patógenos. En este trabajo se evaluó el liofilizado de los postbióticos de B. longum, observándose un $99 \%$ de inhibición de E. cloacae en cultivos in vitro. Estos resultados se suman a las diversas investigaciones con probióticos encaminados al tratamiento y prevención de infecciones ocasionadas por $E$. cloacae.

ABSTRACT

Enterobacter cloacae belongs to the proteobacteria phylum (one of the most abundant in the human gut microbiota), Enterobacteriaceae family, Genus Enterobacter and species Enterobacter cloacae. This bacterium is ubiquitous, opportunistic, resistant to antibiotics and responsible for bacteremia and nosocomial infections, this fact make us search for different treatment and alternatives of control. This work proposes a control of E. cloacae growth inhibition, which is achieved by using bacterial metabolites or cell-free supernatants (also known as postbiotics) produced by Bifidobacterium longum (probiotic belonging to the Actinobacteria phylum); several works have shown that relevance growth inhibition of different pathogenic microorganisms. The B. longum postbiotic lyophilisate have shown $99 \%$ of inhibition on in vitro $E$. cloacae cultures. These results add to various investigations with probiotics aimed at the treatments and prevention of infections caused by E. cloacae.

\section{INTRODUCCIÓN}

La resistencia que presentan las bacterias del género Enterobacter a los antibióticos betalactámicos (ampicilina, amoxicilina y las cefalosporinas de 
issn 1665-4412, e-issn 2521-9758

Barrón-González, M. P., Mireles-Rangel, C., Cuéllar-Guevara, F. L., EguiarteLara, D. J., \& Quiñones-Gutiérrez, Y.
20

tercera generación) es inducida por la hiperexpresión de la beta-lactamasa cromosomal (Livermore, 1998).

La OMS (WHO, por sus siglas en inglés) publicó en 2017 la lista mundial prioritaria de bacterias resistentes a los antibióticos para orientar la investigación, el descubrimiento y el desarrollo de nuevos antibióticos contra microorganismos como Acinetobacter baumanii, Pseudomonas aeruginosa y bacterias de la familia Enterobacteriaceae, resistentes a los carbapenémicos, productoras de beta-lactamasa de espectro extendido (BLEE) y bacterias multirresistentes a las cefalosporinas de tercera generación (WHO, 2017).

Las bacterias de la familia Enteriobacteriaceae son responsables de 30 a $35 \%$ de todas las septicemias, más de $70 \%$ de todas las infecciones del tracto urinario e intestinales (Murray, Rosenthal, \& Pfaller, 2006). A la familia de las enterobacteráceas pertenece Enterobacter cloacae, esta bacteria es un bacilo Gram negativo, oxidasa negativo y catalasa positivo, la cual se encuentra como microbiota habitual en el aparato digestivo del humano.

La mayoría de las infecciones en humanos son causadas por E. cloacae, siendo agentes comunes de infecciones asociadas a la atención en salud (IAAS), principalmente bacteriemias, neumonía asociada a ventilación mecánica, infecciones urinarias e intraabdominales complicadas (Mezzatesta, Gona, \& Stefani, 2012); también es común en pacientes inmunocomprometidos, con quemaduras o pacientes con enfermedades de base (Musil, Jensen, Schilling, Ashdown, \& Kent, 2010), como diabéticos, oncogénicos, entre otros.

La terapia antimicrobiana está indicada en la mayoría de las infecciones por Enterobacter, muy resistentes a agentes antimicrobianos que pueden desarrollar resistencia durante la terapia antimicrobiana, por lo que la elección de agentes antimicrobianos apropiados es complicada; generalmente el tratamiento hacia este género de bacterias incluye cefalosporinas de tercera generación, como cetriaxona, ceftazima (Mezzatesta et al., 2012).

En la búsqueda de alternativas terapéuticas para inhibir la proliferación de microorganismos patógenos al humano se ha recurrido al estudio de una gran diversidad de microorganismos, principalmen- te bacterias y sus metabolitos, a lo que se le conoce como terapia de interferencia microbiana o Microbial Interference Therapy (MIT) (Falagas, Rafailidis, \& Makris, 2008). Entre los microorganismos empleados para este propósito están los probióticos.

Los probióticos fueron definidos en 2002 como microorganismos vivos que confieren beneficios a la salud del huésped cuando son administrados en cantidades adecuadas. Esta definición fue consensada por el comité de expertos de la Organización para la Agricultura y la Alimentación (Food and Agriculture Organization) de las Naciones Unidas y por la OMS (FAO/WHO, 2002); sin embargo, en 2014 el grupo de expertos reunidos por ISAPP (International Scientific Association for Probiotics and Prebiotics) publicaron el Marco general sobre el uso y alcance apropiados del término probiótico, este consenso indica que se requiere evidencia de un beneficio de salud, ya sea a nivel de grupo o específico de la cepa, según la naturaleza del beneficio. Los probióticos pueden tener diferentes medios de administración, especies objetivo (humanos y animales), poblaciones objetivo, sitios objetivo (intestino y más allá), puntos finales de eficacia y categorías reguladoras. Todos los probióticos deben ser seguros para su uso previsto. Los microbios muertos, los productos y componentes microbianos no están incluidos en la clasificación de probióticos (Hill et al., 2014).

Los productos microbianos producidos por los probióticos reciben el término de postbióticos, metabióticos, biogénicos o metabolitos/SFC (sobrenadantes libres de células), secretados por bacterias vivas o liberados después de la lisis bacteriana (Aguilar-Toalá et al., 2018). Estos subproductos pueden contener ácidos grasos de cadena corta (SCFA, por sus siglas en inglés), enzimas, péptidos, ácidos teicoicos, muropéptidos derivados de peptidoglicanos, endo y exopolisacáridos, proteínas de la superficie celular, vitaminas, plasmalógenos y ácidos orgánicos (Tsilingiri \& Rescigno, 2013). Estos subproductos ofrecen beneficios fisiológicos al huésped al proporcionar bioactividad adicional (Cicenia et al., 2016).

Entre los microorganismos probióticos más comunes con beneficios de salud para los seres humanos destacan los siguientes géneros: Lactobacillus, Bifidobacterium, Saccharomyces, Enterococcus, Streptococcus, Pediococcus, Leuconostoc, Bacillus y la especie Escherichia coli (Fijan, 2014). 
El género Bifidobacterium (figura 1) incluye varias bacterias anaerobias no móviles Gram positivas y algunas cepas se caracterizan por presentar mecanismos de resistencia a las sales biliares, las cuales se usan a menudo como bacterias probióticas, entre las cepas que presentan esta característica están Bifidobacterium infantis, B. adolescentis, B. animalis subsp animalis, $B$. animalis subsp lactis, $B$. bifidum, $B$. longum y $B$. breve. Bifidobacterium junto con otros probióticos se emplean para el tratamiento de estreñimiento (Chmielewska \& Szajews$\mathrm{ka}, 2010)$, la diarrea del viajero (McFarland, 2007), la diarrea asociada a antibióticos (Hempel et al., 2012), remisión de la inflamación intestinal y la colitis ulcerosa moderada (Dylag, Hubalewska-Mazgaj, Surmiak, Szmyd, \& Brzozowski, 2014), enterocolitis necrotizante en recién nacidos (Di Gioia, Aloisio, Mazzola, \& Biavati, 2014), reducción de la diarrea inducida por radiación (Demers, Dagnault, \& Desjardins, 2014), reduciendo el desarrollo de riesgo de enfermedad por eccema, alergias a los alimentos (Isolauri, Rautava, \& Salminen, 2012), capacidades para reducir el colesterol (Ruiz, Margolles, \& Sánchez, 2013), alivio de los síntomas de la intolerancia a la lactosa, inmunoestimulación, prevención del cáncer y efectos antialérgicos (Sanders et al., 2013).

Algunas especies de bifidobacterias poseen efectos antagónicos contra patógenos intestinales (Íñiguez-Palomares \& Acedo-Félix, 2006), otras especies como B. breve y B. infantis inhiben la invasión de Escherichia coli enteropatógena, Yersinia pseudotuberculosis y Salmonella typhimurium en cultivo de células Caco-2 enterocite-like (Bernet, Brassat,

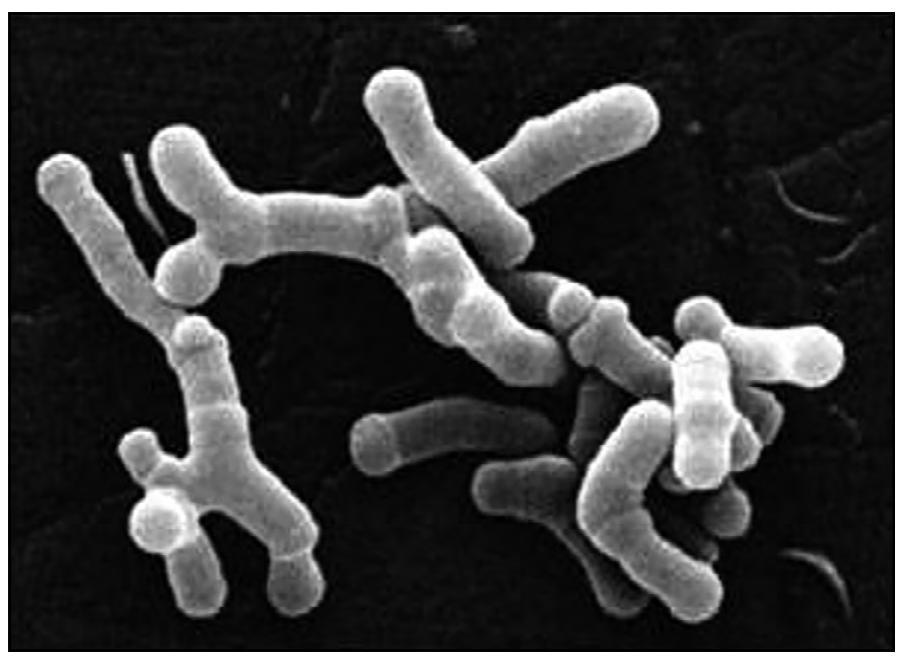

Figura 1. Aspecto morfológico de Bifidobacterium longum. Fotografía de Mark Schell (s. f.).
Nesser, \& Servin, 1993). La inhibición entre los microorganismos ocurre por interferencia bacteriana (IB), la cual es expresada por medio de diversos mecanismos como cambios en el microambiente bacteriano y reducción de los nutrientes o producción de sustancias antagonistas, como bacteriocinas (González-Martínez, Gómez-Treviño, \& JiménezSalas, 2003).

El objetivo de esta investigación fue evaluar la actividad biológica de liofilizados de los postbióticos producidos por B. longum (LPtbt-BI) sobre el crecimiento de E. cloacae. De acuerdo con los antecedentes mostrados, es posible que alguno de los metabolitos o la sinergia de varios de estos, contenidos en el liofilzado del postbiótico de $B$. longum, puedan interferir en actividades metabólicas vitales de E. cloacae.

\section{MATERIALES Y MÉTODOS}

\section{Origen, cultivo y mantenimiento de las cepas}

Las cepas de B. longum (ATCC:15707) y E. cloacae (ATCC:13047) se adquirieron en la American Type Culture Collection (ATCC). Los cultivos bacterianos se mantuvieron a $4{ }^{\circ} \mathrm{C}$ en el medio específico y fueron cultivadas de manera periódica como se describe a continuación:

a) Para el cultivo de E. cloacae se preparó caldo nutritivo Bioxon de acuerdo con las especificaciones del fabricante, posteriormente se alicuotó en $5 \mathrm{ml}$ en tubos de vidrio Pyrex de $13 \times 100$ $\mathrm{mm}$ con tapón de rosca, se esterilizaron en autoclave a $15 \mathrm{Lb}$ de presión, $121^{\circ} \mathrm{C}$ por $20 \mathrm{~min}$, se dejaron temperar, y una vez que pasaron la prueba de esterilidad se almacenaron a $4{ }^{\circ} \mathrm{C}$ hasta su uso.

b) Para el cultivo de B. longum la cepa fue cultivada en el caldo Man-Rogosa-Sharpe (Difco, Becton Dickinson Co., Sparks, MD, USA) e incubado por $24 \mathrm{~h}$ a $37^{\circ} \mathrm{C}$.

\section{Obtención del postbiótico de B. longum (BI)}

Después de la incubación de $B$. longum se obtuvo el sobrenadante libre de células (postbiótico) y se procedió a centrifugar a 2,500 r. p. m. durante 20 min (IEC CL30 Centrifuge Thermo SCIENTIFIC $®$ ). El material recuperado se filtró mediante membrane Millipore $(0.22 \mu \mathrm{m}, \mathrm{GP}$ Millipore Express §PLUS Membrane, Massachusetts, USA). El material filtrado fue colocado en vasos contenedores de vidrio especiales para liofilizar (LABCONCOB). Los vasos contenedores fueron co- 
issn 1665-4412, e-issn 2521-9758

Barrón-González, M. P., Mireles-Rangel, C., Cuéllar-Guevara, F. L., EguiarteLara, D. J., \& Quiñones-Gutiérrez, Y. nectados al equipo para liofilización (LABCONCO® Catalog 7740020) a una presión de 0.133 mBar y una temperatura de colector de $-40^{\circ} \mathrm{C}$ por aproximadamente 12 h (Barrón González et al., 2008); finalmente se obtuvo el liofilizado del sobrenadante libre de células (postbiótico) de B. longum (LPtb-BI).

\section{Preparación de la solución madre del LPtb-BI}

Bajo condiciones de esterilidad se disolvió a saturación el LPtb-Bl en $50 \mathrm{ml}$ de agua desionizada estéril $\mathrm{pH} 7$, posteriormente esta solución madre se esterilizó por filtración (empleando filtros millipore $0.22 \mu \mathrm{m}$ ), se sometió a prueba de esterilidad. Una vez que la prueba de esterilidad resultó positiva se tomaron las concentraciones a evaluar: 40, 20, 10 y 2 mg/ml.

\section{Determinación de la actividad inhibitoria del LPłb- Bl sobre E. cloacae}

Para determinar la interferencia bacteriana del LPtb$B /$ sobre el cultivo de E. cloacae se empleó la metodología reportada por Barrón González et al. (2008). Cada serie de 12 tubos contenía 40, 20, 10 y 2 mg/ $\mathrm{ml}$ del LPtb-Bl, posteriormente fueron inoculados con $100 \mu \mathrm{L}$ de un cultivo de E. cloacae que presentaba una turbidez comparable con el tubo de 0.5 de la escala de McFarland, inmediatamente se incubaron a $37^{\circ} \mathrm{C}$ por $24 \mathrm{~h}$. Enseguida se realizaron lecturas de absorbancia a $570 \mathrm{~nm}$, se graficaron y analizaron los datos obtenidos, posteriormente a la dosis de LPtb-BI que presentó interferencia bacteriana sobre el cultivo de E. cloacae se le determinaron las UFC/ml a través de un recuento bacteriano en placa (cada bioensayo se realizó en tres eventos independientes por triplicado). Como testigo se empleó ciprofloxacino $[0.1 \mathrm{mg} / \mathrm{ml}$, el control positivo fue el cultivo de $E$. cloacae.

\section{Determinación porcentual de proteínas y ácidos grasos en el LPtb-BI}

A la dosis de LPłb-Bl que presentó mayor inhibición sobre el cultivo de E. cloacae se le realizó la cuantificación de:

a) Proteínas totales de acuerdo con la Norma Mexicana NMX-F-608-NORMEX-2011 (Alimentos-determinación de proteínas en alimentos/ método de ensayo-prueba), también se realizó la cuantificación de proteínas totales mediante el programa para detección de proteínas del equipo Thermo Scientific NanoDrop 2000c Spectrophotometer. Se tomó $1 \mu \mathrm{l}$ de la solución del LPtb-BI [40 mg/ml] y se colocó directamente sobre el pedestal del NanoDrop 200C, poste- riormente el brazo del aparato fue movido para cerrar, enseguida el aparato desplegó la lectura en la pantalla y se registró en la bitácora. Se limpió el pedestal y se repitió el procedimiento en tres eventos independientes por triplicado.

b) También se llevó a cabo la determinación porcentual de ácidos grasos totales de acuerdo con la Secretaría de Salud (26 de junio de 1996) con la Norma Oficial Mexicana NOM-086SSA 1-1994 (Bienes y servicios. Alimentos y bebidas no alcohólicas con modificaciones en su composición. Especificaciones nutrimentales).

La determinación de proteínas y ácidos grasos presentes en el LPtb-Bl se llevaron a cabo en un laboratorio de referencia, acreditado ante la Entidad Mexicana de Acreditación (EMA).

\section{Análisis estadístico}

Se realizó el análisis de los resultados obtenidos empleando el análisis estadístico ANOVA con $P<0.05$ en el paquete de análisis estadístico SPSS versión Windows® 2010.

\section{RESULTADOS}

\section{Determinación de la actividad biológica del LPtb-BI sobre E. cloacae}

En la figura 2(a) se observan células de B. longum en fase logarítmica, las cuales presentan la morfología característica bacilar y con aspecto de "Y", lo cual indica que está en proceso de división (flecha), en la imagen (b) se muestra el aspecto del liofilizado obtenido del postbiótico de B. longum (LPtb-BI).

En la tabla 1 y figura 3 se muestran las UFC/ml de $E$. cloacae obtenidas con las cuatro concentra-

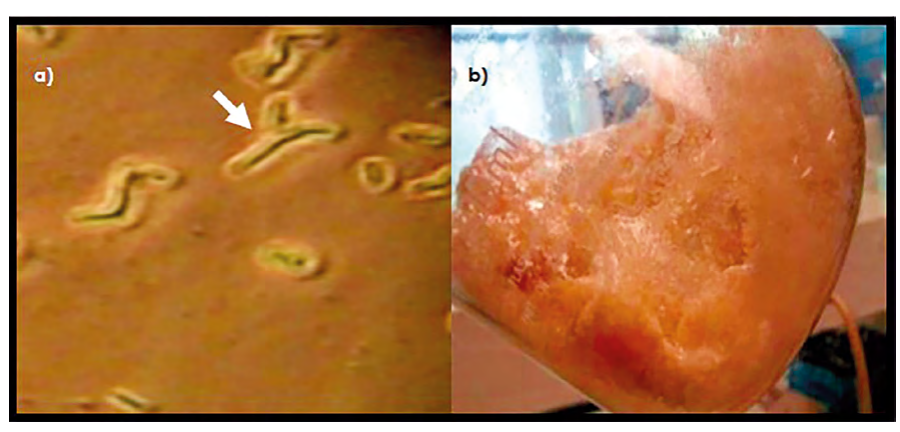

Figura 2. En a) se muestra la morfologia característica de $B$. longum, observada a través de

microscopía de contraste de fase (1000 X), en b) se muestra el aspecto del LPTb-Bl.

Fotografía tomada por María Porfiria Barrón-González. 
Tabla 1

Inhibición del LPtb-BI sobre el crecimiento de E. cloacae

\begin{tabular}{ccc}
\hline Dosis evaluada del LPtb-B.I $(\mathrm{mg} / \mathrm{ml})$ & UFC/ml de E. cloacae $\left(1 \times 10^{6}\right)$ & Inhibición (\%) \\
\hline 40 & 0.907 & 99.9 \\
20 & 2.4 & 99.8 \\
10 & 650 & 44.6 \\
2 & 1105 & 2.8 \\
Ciprofloxacino & 0 & 100 \\
Control & 1173.3 & - \\
\hline
\end{tabular}

Nota: Elaboración propia.

Tabla 2

Determinación de proteínas y ácidos grasos en el LPtb-BI

\begin{tabular}{llc}
\hline $\begin{array}{c}\text { Parámetro determinado en el } \\
\text { LPtb-Bl }\end{array}$ & \multicolumn{1}{c}{$\begin{array}{c}\text { Técnica o metodología } \\
\text { empleada }\end{array}$} & Valor obtenido [mg/ml] \\
\hline Proteínas totales & NMX-F-608-NORMEX-2011 & 22.70 \\
\cline { 2 - 3 } & Nanodrop & 25.25 \\
\hline Ácidos grasos totales & NOM-086-SSA 1-1994 & 7.30 \\
\hline Nota: Elaboración propia. & &
\end{tabular}

ciones evaluadas del LPtb-Bl y el fármaco de elección, asimismo, se muestra el valor porcentual de la inhibición o interferencia bacteriana (IB) que sufrió $E$. cloacae.

En la figura 3 se muestra la inhibición porcentual o la interferencia bacteriana (IB) que exhibe el LPtb-BI sobre la cepa de E. cloacae en concentraciones de 40 y 20 mg/ml y se observó una inhibición porcentual de 99.9 y 99.8 , respectivamente. Estos valores no presentan diferencia significativa con respecto a la inhibición que presentó sobre E. cloacae, el ciprofloxacino [0.1 mg/ml] a esta dosis inhibió $100 \%$ del cultivo. Pero sí presenta diferencia significativa con respecto a la inhibición del cultivo de E. cloacae que se observó al emplear el LPłb-Bl a dosis de 10 y 2 $\mathrm{mg} / \mathrm{ml}$, ya que sólo se obtuvo una inhibición de 45 y $6 \%$, respectivamente.

\section{Cuantificación de proteínas y ácido grasos totales en el LPłb-BI}

En la tabla 2 se muestran los valores obtenidos con respecto a las proteínas totales presentes en el LPtb$B l$, de acuerdo con la NMX-F-608-NORMEX-2011, el valor obteniendo fue de $22.7 \mathrm{mg} / \mathrm{ml}$ y empleando el equipo NanoDrop $25.25 \mathrm{mg} / \mathrm{ml}$, estos valores obtenidos por diferentes técnicas no presentan diferencia significativa. En cuanto a la determinación

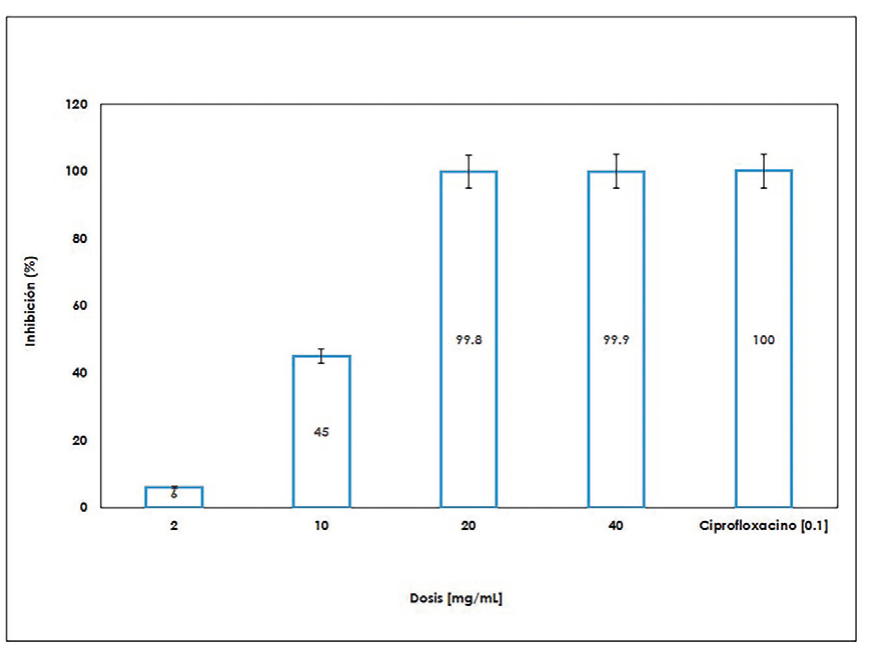

Figura 3. Inhibición porcentual del LPtb-Bl sobre el crecimiento de E. cloacae.

Elaboración propia.

de los ácidos grasos totales, empleando la NOM-086SSA 1-1994, se obtuvo un valor de $7.30 \mathrm{mg} / \mathrm{ml}$.

\section{DISCUSIÓN}

En este trabajo se evaluó el efecto del liofilizado del sobrenadante libre de células (posbiótico) de B. longum (LPtb-Bl) sobre E. cloacae, con mayor 
issn 1665-4412, e-issn 2521-9758

Barrón-González, M. P., Mireles-Rangel, C., Cuéllar-Guevara, F. L., EguiarteLara, D. J., \& Quiñones-Gutiérrez, Y. actividad inhibitoria (interferencia bacteriana) en dosis de 20 y $40 \mathrm{mg} / \mathrm{ml}$, ya que se registró inhibición de 99.8 y $99.9 \%$, respectivamente; de $45 \%$ en dosis de $10 \mathrm{mg} / \mathrm{ml}$; en dosis de $2 \mathrm{mg} / \mathrm{ml}$ fue de $6 \%$, lo cual indica que a mayor concentración de LPtb-BI se observa mayor inhibición del crecimiento de $E$. cloacae.

Al igual que otras bacterias probióticas, B. longum produce sustancias con capacidad bactericida como las bacteriocinas (Moon, Pyun, Park, Ji, \& Kim, 2005), ácidos grasos volátiles de cadena corta, peróxido de hidrógeno y ácido láctico con la consecuente reducción del pH intraluminal; este se considera el principal mecanismo por el cual las bacterias lácticas inhiben el crecimiento de diferentes bacterias patógenas (Donohue \& Salminen, 1996).

Las bacteriocinas son péptidos de síntesis ribosomal que tienen un peso molecular entre 3-10 $\mathrm{kDa}$, pueden presentar actividad antimicrobiana (interferencia microbiana) por diversos mecanismos que incluyen desestabilización de la membrana, lisis celular, degradación de ácidos nucleicos e inhibición de procesos biológicos como síntesis de proteínas, ADN, ARN y peptidoglicano (De-Martinis, Alves, \& Franco, 2002)

En el LPłb-Bl se determinó la presencia de proteínas totales a través de dos técnicas, la NMX-F608-NORMEX-2011 y empleando el NanoDrop 2000c Spectrophotometer, se obtuvieron concentraciones muy semejantes en ambas técnicas; asimismo, se determinaron y cuantificaron los ácidos grasos totales empleando la NOM-086-SSA1-1994, las cuantificaciones mediante especificaciones de las Normas Mexicanas se realizaron en un laboratorio acreditado ante la EMA, ya que asegura una mejor calidad en los resultados.

Según Harmsen et al. (2000), las especies de Bifidobacterium producen un ambiente ácido que evita el crecimiento bacteriano, en este trabajo se demostró la capacidad inhibitoria del postbiótico de B. longum (LPtb-BI), y en otros trabajos se ha demostrado que estas sustancias inhiben el crecimiento de bacterias patógenas por acción antagónica, ya que se bloquea la adhesión al epitelio intestinal (Fuller \& Gibson, 1997).

Se ha reportado que factores extracelulares producidos Lactobacillus acidophilus y L. johnsonii Lal inhiben el crecimiento in vitro de Giardia lamblia en la fase $\mathrm{Gl}$, e impiden que se forme el quiste, la forma infectiva de este protozoario (Pérez et al., 2001). En otro trabajo se reportó la inhibición del ciclo de vida in vitro de Entamoeba histolytica HMlIMSS al emplear liofilizados del sobrenadante de $L$. plantarum, L. casei, L. casei rhamnosus, L. acidophilus y B. longum libre de células (Barrón González et al., 2008).

De acuerdo con los resultados obtenidos en esta investigación, será recomendable realizar la purificación de bacteriocinas producidas por B. longum y evaluar su capacidad para inhibir el crecimiento de E. cloacae y diversos microorganismos patógenos, así como elaborar un perfil proteico de las bacteriocinas para demostrar si el efecto de interferencia bacteriana se debe a una proteína, péptidos o a la sinergia de bacteriocinas $u$ otros metabolitos, y determinar la estructura de las bacteriocinas producidas por B. longum, así como identificar los tipos de ácidos grasos de cadena corta que son liberados por B. longum.

\section{CONCLUSIONES}

En diversos trabajos se han reportado los múltiples efectos benéficos que presentan los probióticos sobre la salud, así como la actividad inhibitoria - de interferencia bacteriana sobre diversos microorganismos patógenos; en esta investigación se observó que el liofilizado del sobrenadante libre de células (postbiótico) de B. longum inhibe el crecimiento de E. cloacae. Estos resultados se suman a las diversas investigaciones con probióticos encaminados al tratamiento y prevención de infecciones ocasionadas por E. cloacae y otros microorganismos patógenos. 


\section{REFERENCIAS}

- Aguilar-Toalá, J. E., García-Varela, R., García, H. S., Mata-Haro, V., González-Córdova, A. F., Vallejo-Córdoba, B., \& HernándezMendoza, A. (2018). Postbiotics: An evolving term within the functional foods field. Trends in Food Science \& Technology, 75, 105-114. doi: 10.1016/j.tifs.2018.03.009

- Barrón González, M. P., Serrano Vázquez, G. C., Villarreal Treviño, L., Verduzco Martínez, J. A., Morales Vallarta, M. R., \& Mata Cárdenas, B. D. (2008). Inhibición del crecimiento axénico in vitro de Entamoeba histolytica por acción de probióticos. Ciencia-UANL, 11, 235-290.

- Bernet, M. F., Brassart, D., Neeser, J. R., \& Servin, A. L. (1993). Adhesion of human bifidobacterial strains to cultured human intestinal epithelial cells and inhibition of enteropathogen-cell interactions. Applied and Environmental Microbiology, 59(12), 4121-4128.

- Chmielewska, A., \& Szajewska, H. (2010). Systematic review of randomised controlled trials: Probiotics for functional constipation. World Journal of Gastroenterology, 16(1), 69-75. doi: 10.3748/wjg.v16.i1.69

- Cicenia, A., Santangelo, F., Gambardella, L., Pallotta, L., lebba, V., Scirocco, A.,... Severi, C. (2016). Protective role of postbiotic mediators secreted by Lactobacillus rhamnosus GG versus Lipopolysaccharide-induced damage in human colonic smooth muscle cells. Journal of Clinical Gastroenterology, 50, S140-S144. doi: 10.1097/MCG.0000000000000681

- De-Martinis, E. C. P., Alves, V. F., \& Franco, B. D. G. M. (2002). Fundamentals and perspectives for the use of bacteriocins produce by lactic acid bacteria in the meat products. Food Reviews International, 18(2-3), 191-208. doi: 10.1081/FRI120014688

- Demers, M., Dagnault, A., \& Desjardins, J. (2014). A randomized double-blind controlled trial: Impact of probiotics on diarrhea in patients treated with pelvic radiation. Clinical Nutrition, 33(5), 761-767. doi: 10.1016/j.clnu.2013.10.015

- Di Gioia, D., Aloisio, I., Mazzola, G., \& Biavati, B. (2014). Bifidobacteria: Their impact on gut microbiota composition and their applications as probiotics in infants. Applied Microbiology and Biotechnology, 98(2), 563-577. doi: 10.1007/ s00253-013-5405-9

- Donohue, D. C., \& Salminen, S. (1996). Safety of probiotic bacteria. Asia Pacific Journal of Clinical Nutrition, 5(1), 25-28.

- Dylag, K., Hubalewska-Mazgaj, M., Surmiak, M., Szmyd, J., \& Brzozowski, T. (2014). Probiotics in the mechanism of protection against gut inflammation and therapy of gastrointestinal disorders. Current Pharmaceutical Design, 20(7), 1149-1155. doi: 10.2174/13816128113199990422

- Falagas, M. E., Rafailidis, P., \& Makris, G. C. (2008). Bacterial interference for the prevention and treatment of infections. International Journal of Antimicrobial Agents, 31 (6), 518-522. doi: 10.1016/j.ijantimicag.2008.01.024

- Fijan, S. (2014). Microorganisms with claimed probiotic properties: An overview of recent literature. International Journal of Environmental Research and Public Health, 11 (5), 4745-4767. doi: 10.3390/ijerph1 10504745

- Food and Agriculture Organization of the United Nations World Health Organization. (2002). Guidelines for the evaluation of probiotics in food. Report of a joint FAO/WHO Working Group on drafting guidelines for hte evaluation of probiotics in food. London Ontario, Canada, April 30 and May 1, 2002. Recuperado el 10 de marzo de 2018, de http://www.who.int/ foodsafety/fs_management/en/probiotic_guidelines.pdf

- Fuller, R., \& Gibson, G. R. (1997). Modification of the intestinal microflora using probiotics and prebiotics. Scandinavian Journal of Gastroenterology, 32(222), 28-31. doi: 10.1080/00365521.1997.11720714

- González-Martínez, B. E., Gómez-Treviño, M., \& JiménezSalas, Z. (2003). Bacteriocinas de probióticos. Revista de Salud Pública y Nutrición, 4 (2). Recuperado de http://www. medigraphic.com/pdfs/revsalpubnut/spn-2003/spn032g.pdf

- Harmsen, H. J., Wildeboer-Veloo, A. C. M., Raangs, G. C., Wagendorp, A. A., Klijn, N., Bindels J. G., \& Welling, G. W. (2000). Analysis of intestinal flora development in breast-fed and formula-fed infants by using molecular identification and detection methods. Journal of Pediatric Gastroenterology and Nutrition, 30(1), 61-67. Recuperado de https://journals. IwW.com/jpgn/Fulltext/2000/01000/Analysis_of_Intestinal_ Flora_Development_in.19.aspx

- Hempel, S., Newberry, S. J., Maher, A. R., Wang, Z., Miles, J. N. V., Shanman, R.,..., Shekelle, P. G. (2012). Probiotics for the prevention and treatment of antibiotic-associated diarrhea: A systematic review and meta-analysis. Journal of the American Medical Association, 307(18), 1959-1969. doi: 10.1001/jama.2012.3507

- Hill, C., Guarner, F., Reid, G., Gibson, G. R., Merenstein, D. J., Pot, B., ... Sanders, M. E. (2014). Expert consensus document: The International Scientific Association for Probiotics and Prebiotics consensus statement on the scope and appropriate use of the term probiotic. Nature Reviews. Gastroenterology \& Hepatology, 11, 506-514. doi: 10.1038/nrgastro.2014.66 
issn 1665-4412, e-issn 2521-9758

Barrón-González, M. P., Mireles-Rangel, C., Cuéllar-Guevara, F. L., EguiarteLara, D. J., \& Quiñones-Gutiérrez, Y.
- Íñiguez-Palomares, C., \& Acedo-Félix, E. (2006). Mecanismos de adhesión al tracto intestinal y anatagonismo de Bifidobacterium. Revista de Salud Pública y Nutrición, 7(2). Recuperado de http://respyn.vanl.mx/index.php/respyn/ article/view/168

- Isolauri, E., Rautava, S., \& Salminen, S. (2012). Probiotics in the development and treatment of allergic disease. Gastroenterology Clinics of North America, 41(4), 747-762. doi: 10.1016/j.gtc.2012.08.007

- Livermore, D. M. (1998). $\beta$-lactamase-mediated resistance and opportunities for its control. Journal of Antimicrobial Chemotherapy, 41 (4), 25-41. doi: 10.1093/jac/41.suppl_4.25

- McFarland, L. V. (2007). Meta-analysis of probiotics for the prevention of traveler's diarrhea. Travel Medicine and Infectious Disease, 5(2), 97-105. doi: 10.1016/j. tmaid.2005.10.003

- Mezzatesta, M. L., Gona, F., \& Stefani, S. (2012). Enterobacter cloacae complex: Clinical impact and emerging antibiotic resistance. Future Microbiology, 7(7), 887-902. doi: 10.2217/ fmb.12.61

- Moon, G. S., Pyun, Y. R., Park, M. S., Ji, G. E., \& Kim, W. J. (2005). Secretion of recombinant pediocin PA-1 by Bifidobacterium longum, using the signal sequence for Bifidobacterial $\alpha$-amylase. Applied and Environmental Microbiology, 71 (9), 5630-5632. doi: 10.1128/AEM.71.9.5630-5632.2005

- Murray, P. R., Rosenthal, K. S., \& Pfaller, M. A. (Eds.). (2006). Microbiología médica (5a. ed., pp. 323-335). España: Elsevier.

- Musil, I., Jensen, V., Schilling, J., Ashdown, B., \& Kent, T. (2010). Enterobacter cloacae infection of an expanded polytetrafluoroethylene femoral-popliteal bypass graft: A case report. Journal of Medical Case Reports, 4,131. doi: 10.1186/1752-1947-4-131
- Norma Mexicana NMX-F-608-NORMEX-2011. Alimentosdeterminación de proteínas en alimentos/método de ensayoprueba. Recuperado el 14 marzo de 2018, de dof.gob.mx/ nota_detalle.php? codigo $=4858024 \&$ fecha $=04 / 08 / 0980$

- Pérez, P. F., Minnaard, J., Rouvet, M., Knabenhans, C., Brassart, D., De Antoni, G. L., \& Schiffrin, E. J. (2001). Inhibition of Giardia intestinalis by extracellular factors from Lactobacilli: An in vitro Study. Applied and Environmental Microbiology, 67(11), 5037-5042. doi: 10.1128/AEM.67.11.5037-5042.2001

- Ruiz, L., Margolles, A., \& Sánchez, B. (2013). Bile resistance mechanisms in Lactobacillus and Bifidobacterium. Frontiers in Microbiology, 4, 396. doi: 10.3389/fmicb.2013.00396

- Sanders, M. E., Guarner, F., Guerrant, R., Holt, P. R., Quigley, E. M. M., Sartor, R. B. ..., Mayer, E. A. (2013). An update on the use and investigation of probiotics in health and disease. Gut, 62 (5), 787-796. doi: 10.1136/gutjnl-2012-302504

- Schell, M. (s. f.). Bifidobacterium longum [Fotografía]. Athens, GA: University of Georgia.

- Secretaría de Salud. (26 de junio de 1996). Norma Oficial Mexicana NOM-086-SSA 1-1994, Bienes y Servicios. Alimentos y bebidas no alcohólicas con modificaciones en su composición. Especificaciones nutrimentales. Diario Oficial de la Federación. Recuperado el 14 de marzo de 2018, de dof.gob.mx/nota_detalle.php?cdigo $=5172062 \& f e c$ ha $=22 / 12 / 2010$

- Tsilingiri, K., \& Rescigno, M. (2013). Postbiotics: What else? Beneficial Microbes, 4(1), 101-107. doi: 10.3920/BM2012.0046

- World Health Organization. (2017). Global priority list of antibiotic-resistant bacteria to guide research, discovery, and development of new antibiotics. Recuperado el 10 de marzo de 2018, de http://www.who.int/medicines/publications/ global-priority-list-antibiotic-resistant-bacteria/en/ 